\title{
Optimal Sensor Placement Based on Bisect K-means Clustering Algorithm
}

\author{
Zhaolan Wei $^{1, \text { a }}$, Jing Xia ${ }^{2, b}$ \\ ${ }^{1}$ College of Civil Engineering, Sichuan Agricultural University, Dujiangyan, Sichuan,611830, \\ China. \\ ${ }^{2}$ College of Civil Engineering, Sichuan Agricultural University, Dujiangyan, Sichuan,611830, \\ China. \\ aweifei0722@163.com, ${ }^{b}$ xia2273789637@163.com
}

Keywords: Sensor, K-means clustering algorithm, measurement point

Abstract: Sensor placement is a combinatorial optimization problem. Considering the number of factors, the selection of measuring points is easy to cause information redundancy and low signal-to-noise ratio. In order to solve this problem, according to the matrix of structure's frequency response, bisect K-means clustering algorithm is designed to classify the degrees of freedom according to the similarity of response. This method is applied to the steel truss arch bridge with the background of Nanjing Dashengguan Yangtze River Bridge. The results show that the proposed method in this paper can better classify the degrees of freedom with similar vibration characteristics, make the sensor more balanced in the overall structure, overcome the redundant information among the sensors, and improve the signal-to-noise ratio at the measurement point.

\section{Introduction}

Optimal sensor placement has received much attention over the years because of its importance role in many areas, such as system identification [1], vibration and noise control[2], model updating[3], structural health monitoring[4] and other fields. In the process of data acquisition, due to the limitation of test conditions, it is impossible to place sensors in all degrees of freedom. Therefore, how to place a limited number of sensors in the best position to obtain reliable and comprehensive modal test information has important practical significance.

\section{Background Theory}

\subsection{Modal Assurance Criterion}

The larger space angles among the measured modal vectors should be guaranteed while choosing measuring points in order to keep the original properties of the structure if possible. Carne and Dohmann thought that modal guarantee matrix MAC is a good tool to evaluate the intersection angle of modal vector space, and It can be expressed as[5]: 


$$
\operatorname{MAC}_{i j}=\frac{\phi_{i}^{T} \phi_{j}}{\left(\phi_{i}^{T} \phi_{i}\right)\left(\phi_{j}^{T} \phi_{j}\right)}
$$

Where, $\phi i$ and $\phi j$ represent the ith and jth column vectors in matrix $\phi$. The element values of the $M A C$ matrix range between 0and 1 , When measuring points are selected, the non-diagonal elements of MAC matrix should be minimized. In a more complex structure, the non-diagonal element of MAC matrix can be maximally $0.25[6]$.

\subsection{Root Mean Square Error Criteria}

Based on the output value of the sensor and the cubic spline fitting, the mode shapes of the steel truss beam and the steel truss arch is fitted, and then compared with the calculated values of the finite element to judge the advantages and disadvantages of the layout Scheme. RMS defined as:

$$
R M S_{i}=\left[\frac{1}{N} \sum_{j=1}^{N}\left(\Phi_{i j}^{C S}-\Phi_{i j}^{F E}\right)^{2}\right]^{\frac{1}{2}}
$$

Where, $N$ is the candidate measuring point, and the superscripted $C S$ and $F E$ are the signs of the fitted mode shape and the finite element calculation mode respectively. Generally, the ratio of RMS value to mode amplitude is taken as the relative root mean square error. If the ratio is within $5 \%$, the result that the fitted mode agree with the calculation mode perfectly. If the ratio is between $5 \%$ and $10 \%$, it means good[7].

\subsection{Frequency Response Functions}

The frequency response function $(F R F)$ is the ratio of the structural output response and the input excitation force, reflects the relationship between the system input and output, and represents the inherent properties of the system. Assuming an n-degree-of-freedom damping system, its differential motion equation is:

$$
M \ddot{x}(t)+C \dot{x}(t)+K x(t)=f(t)
$$

In which $M, C$ and $K$ are the mass, damping, and stiffness matrices, respectively, $x(t)$ is the vector of physical displacements, and $f(t)$ is the vector of external forces. Assuming that the system is subjected to a harmonic excitation, the force and displacement response can be expressed as: $f(t)=F e^{j \omega t} ; x(t)=X e^{j \omega t}$. Bring it to Eq. (3), Eq. (3) can be expressed as the following:

$$
\left(K-\omega^{2} M+\mathrm{j} \omega \mathrm{C}\right) X(\omega)=F(\omega)
$$

$H(\omega)=(-\omega 2 M+j \omega C+K)^{-1}$ is the frequency response function, When the harmonic excitation is applied in the ith, the acceleration response at the $j$ th is expressed as follows:

$$
H_{i j}(\omega)==\sum_{r=1}^{N} \frac{-\omega^{2} \phi_{i r} \phi_{j r}}{\omega_{r}^{2}-\omega^{2}+j 2 \zeta_{r} \omega_{r} \omega}
$$

In which $\omega_{r}$ is the undammed natural frequency of a system, $\zeta_{r}$ is the damping coefficient, $\phi_{i r}$ is the value of the rth mode at the ith output location, and $\phi_{j r}$ is the value of the rth mode at the jth input location. 


\subsection{Bisect K-means Clustering Algorithm}

In the process of structural vibration, some degrees of freedom contain similar vibration characteristics, and these response of the degrees of freedom is approximately equal at a certain load. The bisect K-means clustering algorithm classifies these degrees of freedom automatically based on the similarity of multiple attribute.

$X=\left\{x_{1}, x_{2}, \ldots x_{n}\right\}$ be the data of n-dimensional space. First, all points are taken as a cluster and then divided into two clusters. After that, the cluster that can minimize the clustering cost function (that is, sum of squared error) are divided into two clusters, and the process continues until the number of clusters is equal to the user-given number $\mathrm{k}$. This is the main idea of the bisect K-means clustering algorithm. The indicator for evaluating the effectiveness of clustering is the sum of squared error (SSE), which is defined as[8]:

$$
S S E=\sum_{i=1}^{k} \sum_{x \in C_{i}}^{m}\left(x-c_{i}\right)^{2}
$$

Where: $c_{i}$ is the cluster center of the cluster $C_{i}$ and $\mathrm{x}$ is a sample in the cluster The steps of sensor placement are as follows:

(1) Establish a finite element model and extract modal vectors based on the mode shape characteristics.

(2) According to the modal confidence criterion, make the relationship between the maximum value of non-diagonal MAC matrix and the number of measurement points to determine the number of sensors.

(3) Using the extracted modal vectors, calculate the matrix of acceleration frequency response function according to the above formula.

(4) The matrix of frequency response function is used as a sample, and the bisect k-means algorithm programmed by MATLAB is used to divide the degrees of freedom into k clusters and then select the nearest point of the cluster center to arrange the sensor.

\section{Engineering Applications}

\subsection{Introduction to the bridge}

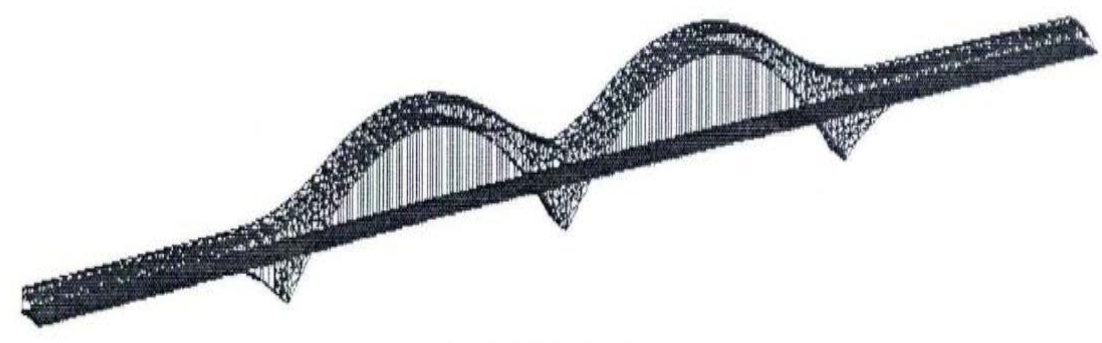

Figure 1. FEM model of the bridge.

The major part of the Nanjing Dashengguan Yangtze River Bridge is symmetrically arranged with 108+192+336+336+192+108 m six-span continuous steel trussed-arch structure. The deck is $41.6 \mathrm{~m}$ in width, which consists of the center-to-center distance between the three main trusses is $15.0 \mathrm{~m}$ and the two sides of the cantilevered decks with a width of $5.8 \mathrm{~m}$. The $2 \times 336 \mathrm{~m}$ main arches are steel structure, the arch-rise is $84.2 \mathrm{~m}$, corresponding to the rise-to-span ratio of 1:4. Next to both sides of the main arches are $\mathrm{N}$-shaped flat chord trusses, which are $240 \mathrm{~m}$ in length and $16 \mathrm{~m}$ in height, with a panel length of $12 \mathrm{~m}$ for most panels. The deck of the bridge is designed as a 
monolithic orthotropic steel plate stiffened. The bridge has 11775 nodes and 19364 units, the FEM model of the Nanjing Yangtze River Bridge is shown in Figure 1. The descriptions for the first 10 modes are given in Table 1.

Table 1. Modal Properties of the bridge.

\begin{tabular}{crc}
\hline Numble & Frequency/Hz & Mode description \\
\hline 1 & 0.3735 & 1st vertical anti-symmetry. \\
2 & 0.4557 & 1st lateral bend anti-symmetry with arch and \\
3 & 0.4774 & girder in phase. \\
4 & 0.6844 & lateral symmetry with arch and girder in phase. \\
5 & 0.6883 & 2st vertical anti-symmetry. \\
6 & 0.7972 & 3nd vertical symmetry. \\
7 & 0.8097 & Reverse anti-symmetry bends, twisting. \\
8 & 0.8616 & Reverse symmetry bends, twisting. \\
\hline
\end{tabular}

\subsection{Optimal sensor placement}

The first 8 vertical modes of the structure are selected to form a modal vector. Due to the symmetrical structure of the bridge, half of the truss structure in the middle were used for analysis. In order to avoid the mixture of girder arch information, the girder and arch are separated to optimize the sensor.

\subsubsection{Determination of the number of sensors}

This paper uses the maximum value of non-cross element MAC matrix and the number of points to determine the number of measurement. In Figure. 2, when the truss girder is arranged two sensors, the maximum non-cross element is 0.9461 , and 10 sensors is 0.128 . After increasing the point, it does not change significantly, so we choose to arrange 10 sensors. For the arch part, when the 5 sensors are arranged, the maximum non-cross element is 0.2229 . After continuing to increase the point, the curve descends very slowly, so the arch rib is arranged with 5 sensors.

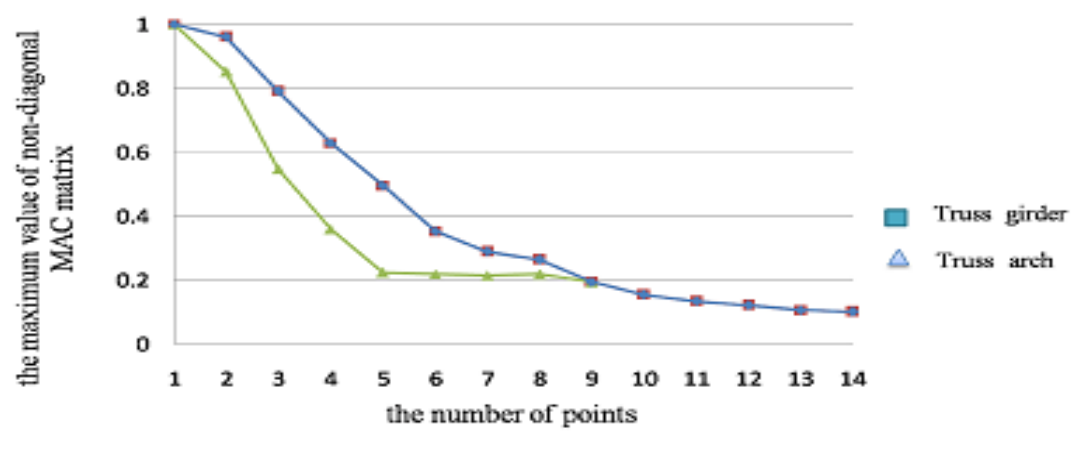

Figure 2. Change of non-diagonal elements with the number of points

\subsubsection{Sensor position determination}

The modal vector is normalized, and the frequency response matrix is calculated according to the above formula. The bisect k-means algorithm programmed by MATLAB, which is used to divide the girder degrees of freedom into 10 clusters and the arch into 5 clusters. The nearest point in each 
cluster is selected as the measuring points. The measurement points of the truss girder are E5, E12, E18, E25, E28, E32, E38, E44, E48, E51and the arch are S29, S33, S38, S43, S47.

\subsection{Results Analysis}

According to the modal vector composed of the above measurement points, the maximal MAC non-cross elements of girder and arch were 0.2335 and 0.1810 , respectively, both less than 0.25 . It shows that the obtained modal information is independent and the information utilization rate is high. The root mean square error calculated by the above formula is shown in Table. 2 . In addition to the 2st mode of truss arch, the root mean square root error of the truss girder and arch is less than $5 \%$, which shows that the expansion mode agrees well with the calculated modes. The 2st vibration mode of the arch is $5.27 \%$, within $10 \%$, the fitting condition is good. Therefore, the layout of the measuring points provided in this paper can achieve the purpose of accurately identifying the mode shapes.

Table 2. Relative RMS Error of the fitted mode and finite element calculations

\begin{tabular}{ccccccc}
\hline NO. & 1 & 2 & 3 & 4 & 5 & 6 \\
\hline Truss gird & $2.47 \%$ & $4.82 \%$ & 3.955 & $2.12 \%$ & $2.34 \%$ & $1.44 \%$ \\
Truss arch & $1.27 \%$ & $5.27 \%$ & $4.67 \%$ & $1.38 \&$ & $1.89 \%$ & $1.73 \%$ \\
\hline
\end{tabular}

\section{Conclusion}

By minimizing the non-cross elements of the MAC matrix, adding a small number of sensors in a reasonable position, the modal test results can be improved to a maximum. The Bisect K-means clustering algorithm can divide the similar freedom degree into one class according to the vibration characteristics, and make the sensor more balanced on the whole structure, overcome the information redundancy between the sensors, and improve the signal to noise ratio of the measurement points. For long-span bridges, the clustering algorithm can classify many degrees of freedom, providing a good candidate for the sensor point selection.

\section{References}

[1] Stephan C. Sensor placement for modal identification[J]. Mechanical Systems \& Signal Processing, 2012 , 27(1):461-470.

[2] Loghmani A, Danesh M, Keshmiri M. Modal structural acoustic sensing with minimum number of optimally placed piezoelectric sensors[J]. Journal of Sound \& Vibration, 2015, 363:345-358.

[3] Batou A. Model updating in structural dynamics - Uncertainties on the position and orientation of sensors and actuators[J]. Journal of Sound \& Vibration, 2015, 354:47-64.

[4] Iliopoulos A, Shirzadeh R, Weijtjens W, et al. A modal decomposition and expansion approach for prediction of dynamic responses on a monopile offshore wind turbine using a limited number of vibration sensors[J]. Mechanical Systems \& Signal Processing, 2016, s 68-69:84-104.

[5] Carne T G, Dohmann C R. A modal test design strategy for modal correlation [C]. Nashville, Tennessee, USA: 1995.

[6] Zhou Z H, Sun J L, Xu L Q, et al. Research on Optimal accelerometer placement for Long-span Continuous Rigid Frame Bridge Health Monitoring[J]. Earthquake Engineering and Engineering Vibration, 2009, 29(2):150-158.

[7] He L J, Lian J J, Ma B, et al. Optimal sensors placement for large space structures based on distance coefficient and effective independence method [J]. Vibration and shock, 2013, 32(16):13-18.

[8] Qiu G Y, Zhang J. Adaptive Classification Method of SVM Decision Tree Based on Bisect K-Means [J]. Application Research of Computers, 2012, 29(10):3685-3687. 